\title{
Correlative Microscopy: One Specimen - Many Microscopies - Lots of Info
}

\author{
Ralph Albrecht
}

Dept. of Animal Sciences, University of Wisconsin, 1675 Observatory Dr., Madison, WI 53706

Correlative microscopy can be performed in parallel or series. The former utilizes several samples of the same material examined in different microscopic modes. The latter uses the exact same specimen examined sequentially in different microscopic modes in the same or in different microscopes. The series approach is perhaps the more powerful of the two due to the direct correlations that can be made and here we will deal primarily with this technology [1]. However the parallel approach can also provide valuable correlative information and should not be discarded out of hand.

A typical starting point is the living cell. Often studies must determine dynamic changes in surface and intracellular structure, movement of surface molecular species, changes in cytoskeletal organization, cell to cell contacts, cell to substrate contacts, cell migration, and information on a variety of other parameters. For these studies it is important to consult the modulation transfer function of various microscopic modes to determine which mode is best for the size and nature of the cell or sub-cellular structure of interest [2]. Interference based systems such as Phase Contrast, Differential Interference Contrast, DIC, or Asymmetrical Illumination Contrast, AIC, in combination with sensitive CCD cameras provide a wealth of information about living cells based on intrinsic form and/or composition birefringence without the necessity to stain or label the cells. Using wide field microscopy, lower intensity and longer wavelength light, and typical deconvolution software gives one the ability to view cells over long periods of time and extract information at the multicellular, cellular, and sub-cellular levels of resolution. It is important to be able to select sequentially from several microscopy modes such that the mode of imaging is optimized for size and nature of each of the structures being observed. Interference Reflectance imaging can be specifically used to follow cell substrate contacts in stationary or motile cells. This can be in concert with previously mentioned interference modes. Multi photon or confocal microscopy (spinning disc or slit-scan) can be of advantage where confocal imaging of multicellular structures is necessary.

Labeling of living cells provides substantial additional information although effects of the labeling have to be considered. Fluorescence labeling is perhaps the most widely used method. Cellgenerated label such as GFP and similar variations which now include smaller fluorescent molecules, is an effective way of tracking movement of cells or specific molecular species within cells. External labels can also be applied for surface labeling or to follow binding and internalization of labeled species. Methods of enhancing label uptake or permeablization of cells via methodology which are compatible with cell membrane repair and recovery of cell function can be employed. Various fluorescent dyes which can be used to determine location and quantity of intracellular ionic species such as calcium can be used in conjunction with interference based imaging. With suitable cameras, filters, and beam splitters changes in structure and molecular organization can be correlated directly and simultaneously with changes in cytosolic free ion levels. If small colloidal heavy metal particles of 10nm (molecular size range) and above are conjugated to specific antibodies or ligands, the movement of individual ligand-colloid complexes (presumably following their attachment to the specific receptor or antigen) can be followed over time. The small particles are well below the resolving limits of the light microscope but can be tracked via their inflated diffraction image [3]. It has to be kept in mind that labeling of living cells with ligand or antibody or with active fragment of 
ligand or antibody may require labeling parameters which differ substantially from those used for fixed inanimate material. High efficiencies of labeling must often be attained over very short time periods and nonspecific labeling must be limited [4].

Following observation in the living state, cells can be fixed and stained by a variety of conventional chromatic stains, fluorescent stains, or opaque stains (precipitates or conjugated metal particles). These stains provide additional information on structure and localization of cellular and intracellular elements using wide field and confocal imaging modes [1,3]

In all cases we have found it possible to modify conventional protocols to insure that the cells remain hydrated and in a suitable ionic environment to maintain surface and internal ultrastructure so that cells, studied via light microscopy, can be prepared for subsequent observation via electron based and force based imaging modes. In this way the exact same cell or cells and even the exact same individual colloidal particle label that was tracked in a living cell with LM can subsequently be observed at much higher levels of resolution via SEM \&/or TEM \&/or AFM instrumentation [1,3]. In order to correlate imaging of individual cells or specific molecules within cells accurate marking of cells is critical. This permits relocation of the area of observation in the different instruments. We have employed a number of strategies which range from simple patterns scribed by hand or mechanically on glass or plastic substrates to various commercially available "finder" grids filmed with polymers such as formvar or polystyrene (protein coated or uncoated). Specially formed marking patterns via lithographic methodology and electronic microchip/micromachine fabrication technology have also been employed depending on the sample and information required.

Studies can move directly from the living cell either via chemical fixation usually a formaldehydeglutaraldehyde mix or via rapid physical fixation, (slush nitrogen, high pressure freezing, or, for those who value life a bit less, liquid propane, etc). Preparation of samples is per conventional methodology and frozen hydrated, freeze dried, freeze substituted (followed by CPD, freeze drying, or cold temperature embedding) specimens all can be prepared. For chemically fixed specimens a wide variety of techniques can be used including freeze drying, dehydration via the critical point procedure and any number of embedding procedures $[1,3]$. Whole mount preparations are perhaps the best candidates for series correlative microscopy. Studies which require subsequent subdivision of the specimen, such as via ethanol cryofracture, or sectioning of frozen or embedded material, put further constraints on the identification/relocation method. However when suitable care is taken, one for one correlation is attainable between the various microscopic methodologies $[3,5,6]$.

Currently we are developing correlative labeling methodologies using colloidal particles of different composition and/or shape to permit multiple labeling and co-localization at the molecular and submolecular level of resolution. The methodology permits correlative interference based and/or fluorescent labeling in LM studies on living cells which then can be directly correlated, cell for cell, label for label, with high resolution imaging via FESEM, EFTEM, and AFM [5,6].

\section{References}

[1] R.M. Albrecht and B.K. Wetzel, JMSA Procedings Microscopy and Microanalysis (1995)684

[2] S. Inoue, Video Microscopy, Plenum, New York, 1986.

[3] R.M. Albrecht et.al., in Immunochemistry a Practical Approach, Oxford. Press. (1993)151.

[4] K. Park et. al., in Colloidal Gold Vol 1. Academic Press, New York, (1989). 489

[5] R.M. Albrecht and D.A. Meyer, Micros.Microanal. 8 (suppl.2) (2002) 194.

[6] E. Rosa-Molinar et.al., Micros. Microanal. 8 (suppl.2) (2002) 132.

[7] Supported in part by NIH/NIGMS \#63001 